\title{
Facial Recognition Based on Discrete Wavelet Transform and Component Analysis Support Vector Machine
}

\author{
Jiangxiong $\mathrm{ZHU}^{1, \mathrm{a}}$ and Chang $\mathrm{FENG}^{2, \mathrm{~b}}$
}

${ }^{1}$ School of Physics And Electronic Engineering of Leshan Normal university, Leshan 614004, China

${ }^{2}$ School of Physics And Electronic Engineering of Leshan Normal university, Leshan 614004, China 29265599@qq.com , 14987625 @ qq.com

\begin{abstract}
Keywords: Facial recognition; discrete wavelet transform; independent component analysis; kernel function support vector machine

Abstract. In order to realize facial recognition with different characters such as illumination, posture and noise and improve the recognition precision, a facial recognition method based on discrete wavelet transform and least squares support vector machine is proposed. the discrete wavelet transform is used to compress the facial figure and reducing the noise to get the character information component with low frequency, and then the fast independent component analysis is used to obtain the facial character information with low frequency to reduce the dimension further. Finally, the radius basis function is used as the kernel function, and the training data is input to the least squares support vector machine to get the final recognition model. The simulation experiment is simulated in ORL database with Matlab tool, and the result shows the method in this paper can realize the facial recognition.
\end{abstract}

\section{Introduction}

Facial recognition is a research focus in the field of pattern recognition and computer vision, which has a wide application prospect in the field of security detection system, file management and human-computer interaction etc[1].

The extraction of facial feature is primary problem in facial recognition. At present, based on the locality preserving projection, neural network, support vector machine, $\mathrm{K}$ mean and linear discrimination are the mainstream recognition methods[2-3].

The References [4] had designed the maximum component value discrimination method, threshold classification method and center region classification feature classification of the BP neural network to feature classification and facial recognition.

The References [5] designed a face recognition method based on NMF matrix.In the paper, firstly, the low frequency information of face samples was obtained by discrete wavelet transform, then, the base sample matrix and the projection were calculated by NMF, at the same time, the main feature was extracted by threshold judgement, finally, the facial data was classified by the support vector machine.

The References [6] extracted the invariant component of the face Lambertian model by the soft threshold denoising method,then calculated the Zemike moments as feature vector classification, finally classified face data by the nearest neighbor K-mean.

The above method had important significance, The paper proposed to facial recognition based on discrete wavelet transform and principal component analysis support vector machine.

\section{Discrete Wavelet Transform Denoising}

Suppose: $L^{2}(R)$ :the real numbers space of square integrable, $\varphi(w)$ :Fourier transform,if $\varphi(w)$ met the permit conditions:

$$
C_{\varphi}=\int_{R} \frac{|\varphi(w)|^{2}}{|w|} d w<\infty
$$

Above the formula,the $\psi(t)$ was the mother wavelet, we can get 1 consecutive wavelet sequence by drawing or translating it, as shown below: 


$$
\psi(t)=\sqrt{a}\left(\frac{t-b}{a}\right)
$$

Above the formula, a was the scale factor, $b$ was the translation factor, One dimensional continuous wavelet transform can be defined as:

$W(a, b)=\int_{-\infty}^{\infty} f(t) \frac{1}{\sqrt{a}} \psi\left(\frac{t-b}{a}\right)$

The formula (3) scale and displacement according to the power of 2 discretization, we can get the wavelet discretization, so one-dimensional wavelet discretization can be defined as:

$<f, \psi_{m, n}>=a_{0}^{-m / 2} \int_{-\infty}^{\infty} f(t) \psi\left(a_{0}^{-m} t-n b_{0}\right) d t$

The two-dimensional discretization wavelet transform was composed of two dimensional scaling function $\varphi(x, y)$, the two-dimensional wavelet function $\varphi^{H}(x, y)$ of horizontal direction, the two-dimensional wavelet function $\varphi^{H}(x, y)$ of vertical direction and the two dimensional scaling function of diagonal direction. The discrete wavelet transform for the image $f(x, y)$ whose scale was $N \times M$ can be expressed as:

$W_{\psi}\left(j_{0}, m, n\right)=\frac{1}{\sqrt{M N}} \sum_{x=0}^{M-1} \sum_{y=0}^{N-1} f(x, y) \psi_{j_{0}, m, n}(x, y)$

Above the formula, $W_{\psi}\left(j_{0}, m, n\right)$ Coefficient expressed approximation of $f(x, y)$ in scales $j_{0}$,then for any scale $i \in\{H, V, D\}, W_{\psi}\left(j_{0}, m, n\right)$ expressed detail information of the horizontal direction, the vertical direction and the diagonal direction in the $j \geq j_{0}$. Which can be expressed as:

$$
W_{\psi\left(j_{0}, m, n\right)}^{i}=\frac{1}{\sqrt{M N}} \sum_{x=0}^{M-1} \sum_{y=0}^{N-1} f(x, y) \psi_{j, m, n}^{i}(x, y)
$$

\section{Extract the Feature Vector of Independent Component}

After discrete wavelet transform for denoising, the feature vector of original image was extracted. As an effective blind BSS analysis, ICA independent component analysis technique used a set of independent basis functions, which represented a series of random variables, The model was as follows:

$$
X=A S+\eta
$$

Above the formula, $x$ said the m-dimensions observed signals, A was an unknown mixing matrix of $m \times n, s$ was an unknown signal source for each independent component. $\eta$ was the noise. When A and $S$ were unknown, the main task of ICA independent component analysis was to get the separation matrix $W=A^{-1}$ according to certain rules, Then the output of $Y$ was the optimal approximate estimation of $s$. As shown in the formula (8) and Figure 2:

$Y=W x$

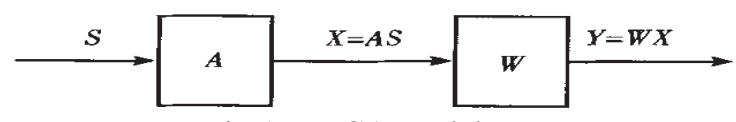

Fig 1 ICA model

Using the fast ICA method, the ${ }^{w_{i}}$ of the $i$ line in the separate the matrix $W$ can be expressed as: 
$w_{i}=E\left\{z \tanh \left(w_{i}^{T} z\right)\right\}-E\left\{\tanh \left(w_{i}^{T} z\right)\right\} w_{i}$

Above the formula, ${ }^{w_{i}^{T}}$ was the transposition of $w_{i}$ components.

Face image feature extraction using ICA independent component method can be expressed as:

(1) Through the rows or columns overlap ,the as input matrix X was constituted by the original face image;

(2) For the characteristic value of matrix from matrix D and the feature vector matrix E, then the data was processed;

$X^{\prime}=X-E(X)$

$Z=D^{-1 / 2} E^{T} X$

(3) Using the formula (11), independent component analysis was applicated in the $Z$, The output matrix $Y$ who was independent of each other based image in space was calculated. each line in the $\mathrm{Y}$ is the independent face based image.

\section{Face Recognition Based on Support Vector Machine}

Least squares support vector machine (LSSVM) has a strong advantage in small sample, nonlinear and high dimensional data. By introducing the kernel function, the linear non separable problem in low dimensional space can be transformed to the inner product operation in high dimensional space by LSSVM.LSSVM model as shown in Figure.

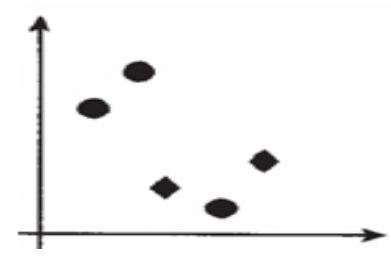

Low dimensional sample space

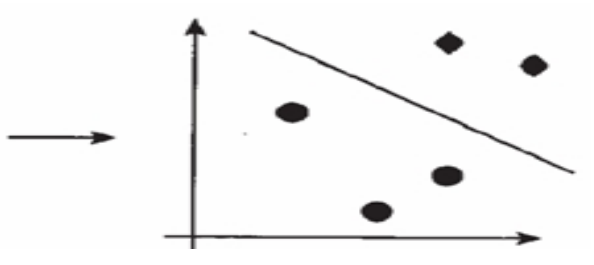

High dimensional sample space

Fig 2 LSSV Model

Assuming: $R^{N}$ was the low dimensional space, $H$ was the high dimensional kernel space, $\phi(x): R^{N} \rightarrow H, x^{\prime}=\phi(x)$ was the nonlinear mapping function, then the optimal hyperplane which was established in the high dimension space can be expressed as:

$w^{T} \phi(x)+\theta=0$

Above the formula, $w$ was the method vector of hyperplanes, $\theta$ was the eviation of hyperplanes.

So the classification problem was transformed into solving the target function in the formula (13). On the formula (13) using the Lagrange multipliers method can obtain the formula (14):

$$
\begin{aligned}
& \left\{\begin{array}{l}
\mathrm{m} \text { in } \quad \frac{1}{2} w^{T} w+\frac{1}{2} c \sum_{i=1}^{N} \xi_{i}{ }^{2} \\
\text { s.t. } y_{i}=w^{T} \phi\left(x_{i}\right)+\theta+\xi_{i} \quad 1 \leq i \leq N
\end{array}\right. \\
& L(w, \theta, \xi, \alpha)=\frac{1}{2} w^{T} w+\frac{1}{2} c \sum_{i=1}^{N} \xi_{i}{ }^{2}- \\
& \sum_{i=1}^{N} \alpha_{i}\left\{y_{i}\left[w^{T} x_{i} \phi\left(x_{i}\right)+\theta\right]-1+\xi_{i}\right\}
\end{aligned}
$$


Above the formula, $\alpha_{i}$ was the lagrange operator, $c$ was the regularization parameter,

$\xi_{i}$ was the slack variables, according to the KKT (Karush-kuhn-Tucker) condition, the objective function can be expressed as:

$f(x)=\operatorname{sign}\left(\sum_{i, j=1}^{m} a_{i} y_{i} k\left(x_{i}, x_{j}\right)+\theta\right)$

Above the formula, sign was the basic sign function. Therefore, through the 1 or -1 of the $f(x)$,we can distinguish two types of samples. $k\left(x_{i}, x_{j}\right)$ was the kernel function.

Because the 1 LSSVM can only carry 2 classification, in order to realize the $n$ classification, the $n(n-1) / 2$ LSSVM need be constructed, and each LSSVM classification results need be voted, according to the principle of the minority obeying the majority, the classification result which was the largest number of votes to was used as the final result.

\section{The Simulation Experiment}

To verify the method in the paper,we used Matlab simulation tools. The experimental samples were from the ORL face database which was composed of 400 facial image,each which was the 112 *92 and gray level composition of 256. a total of 40 people, each had 10 images of different face images, these expressions were composed of anger, disgust, fear consists mainly of joy, sadness and surprise, neutral, etc. 6 pairs of 10 images of different face images as training sample set, the other 4 image as a test sample set, the classification of training samples of 40 , part of the sample in the ORL face database were as follows:

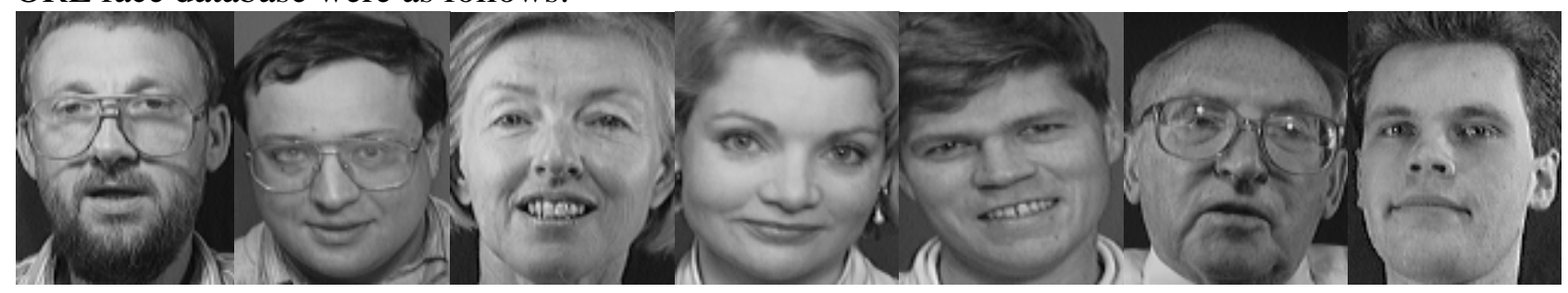

Fig 3 Part of the ORL face sample data

Firstly, the face image of low-frequency characteristics and denoise were obtained by discrete wavelet transform, then we got the feature vector of face image by using ICA component analysis method, at last, radial basis kernel function $k\left(x_{i}, x_{j}\right)=\exp \left\{-g\left\|x_{i}-x_{j}\right\|^{2}\right\}$ was used as kernel function, We trained sample data which was input to the LSSVM so as to get the final classification model. At this time, the input of test samples were for validation, repeated 8 times of experiment, and compared with the literature [6] and [7], the results were as follows: 
TABLE 1 COMPARISON OF RATE OF FACE RECOGNITION

\begin{tabular}{cccc}
\hline \multirow{2}{*}{$\mathrm{t}$} & \multicolumn{3}{c}{ rec ognition rate \% } \\
\cline { 2 - 4 } imes & Reference & Reference & the method in this \\
& $\mathrm{s}[6]$ & $\mathrm{s}$ [7] & paper \\
2 & 93.15 & 95.45 & 98.34 \\
3 & 91.45 & 96.89 & 99.11 \\
4 & 93.64 & 93.87 & 98.53 \\
5 & 94.12 & 97.75 & 99.34 \\
6 & 94.34 & 95.32 & 98.64 \\
7 & 95.64 & 97.45 & 99.01 \\
8 & 92.97 & 95.23 & 99.14 \\
\hline
\end{tabular}

It was seen from table 1 , in the simulation period, the rate of face recognition in this paper methods was significantly higher than that of references [6] and [7]. In this paper, the average recognition rate was as high as $98.92 \%$, the rate of face recognition in references [6] was $94.03 \%$, the rate of face recognition in references [7] was 96.15\%, Obviously, the method in this paper was better.

\section{Conclusion}

In order to realize facial recognition, a facial recognition method based on discrete wavelet transform and least squares support vector machine was proposed. Firstly, the two-dimensional discrete wavelet transform was used to reduce the noise and remove high dimensional components to obtain the facial character information with low frequency, and then the fast independent component analysis is used to to reduce the dimension further. Finally, the radius basis function was used as the kernel function, and the training data was input to the least squares support vector machine to get the final recognition model. The simulation experiment was simulated in ORL database with Matlab tool, and the result showed the method in this paper had high recognition accuracy, which was a effective and feasible method for face recognition.

\section{References}

[1] Yan Juan, Cheng Wushan, Sun Xin. Research and Prospect of face recognition technology[J]. video engineering. 2006(12):81-84

[2] Jiang Wentao, Liu Wanjun, Yuan Da. Facial recognition Based on spatial curved field[J].Chinese Journal of computers, 2012,8(35):1740-1750.

[3] Hu Fengsong, Zhang Maojun, Zou Beiji,Ma Junrong. Facial recognition Based on the Single sample illumination of HMM and pose face[J] . Chinese Journal of computers, 2009,7(32):1424-1433.

[4] Wen Zhou, Shao Xiaowei, Gong Deren. Facial recognition classification Based on BP neural network[J].JOURNAL OF COMPUTER APPLICATIONS, 2011,31(2):133-136.

[5] Lin Qing, Li Jia, Yong Jianping, Liao Dingan. Facial recognition Based on the Improved NMF[J].COMPUTER SCIENCE, 2012,5(39):243-246.

[6] Liu Xiaoshan,Du Minghui, Ceng Chunyan, Jin Lianwen. Facial recognition based on NSCT and pseudo Zemike moments[J]. Journal of South China University of Technology (NATURAL SCIENCE EDITION) ,2011,7(39):82-87.

[7] Lu Guifu, Lin Zhong, Jin Zhong. Optimal discriminant analysis based on kernel extension of graph embedding and face recognition [J]. Journal Of Software, 2011, 22(7): 1561-1570 